\title{
TABLE OF CONTENTS / TABLE DES MATIÈRES
}

Acknowledgments/Remerciements vii

Introduction ix

THOMAS SCHMIDT ET PASCALE FLEURY

\section{SECTION I: ESSENCE ET PRÉSENCE DE LA SECONDE SOPHISTIQUE 1}

Narrative of Cultural Geography in Philostratus's

Lives of the Sophists 3

ADAM KEMEZIS

The Second Sophistic and Non-Elite Speakers 23 IAN HENDERSON

L'Héraclès d'Hérode: héroïsme et philosophie dans la sophistique de Philostrate 36 DOMINIQUE CÔTÉ

\section{SECTION II: L'ORATEUR ET SON IMAGE 63}

L'orateur oracle: une image sophistique 65 PASCALE FLEURY

Portrait d'un rhéteur: Aelius Aristide comme initié mystique et athlète dans les Discours sacrés 76 


\section{vi Contents}

Une écriture du visuel au temps de la Seconde Sophistique: Clément d'Alexandrie (Protreptique) et Philostrate (Images) 87

ANNE PASQUIER

\section{SECTION III: PASSÉ ET IDENTITÉ GRECQUE 103}

Sophistes, barbares et identité grecque: le cas de Dion Chrysostome 105 THOMAS SCHMIDT

Reflets de l'hellénisme chez Athénée à travers l'emploi des termes

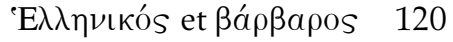

MARIE-HÉLÈNE MAINGUY

Pausanias le Périégète et la Seconde Sophistique 133

JANICK AUBERGER

SECTION IV: TEXTE, TRADITION ET PERFORMANCE 147

Dance and Discourse in Plutarch's Table Talks 9.15 149

KARIN SCHLAPBACH

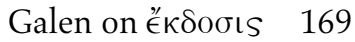

SEAN A. GURD

SECTION V: HÉRITAGE ET INFLUENCE DE LA SECONDE SOPHISTIQUE 185

Were the Speeches of Aelius Aristides 'Rediscovered' in the 350s p.C.? 187

JOHN VANDERSPOEL

Libanius's Monody for Daphne (Oration 60) and the Eleusinios Logos of Aelius Aristides 199 DIANE JOHNSON

Thémistios et la Seconde Sophistique: le thème du tyran 216 CHRISTIAN R. RASCHLE

Bibliography/Bibliographie 235

Index Locorum 259

Index of Proper Names/Index des noms propre 267

Subject Index/Index thématique 270 